\title{
Peripheral neuropathy in hepatitis $C$ virus infection with and without cryoglobulinaemia
}

\author{
R Nemni, L Sanvito, A Quattrini, G Santuccio, M Camerlingo, N Canal
}

J Neurol Neurosurg Psychiatry 2003;74:1267-1271

See end of article for authors' affiliations .....................

Correspondence to: Professor R Nemni, Department of Neurorehabilitation, IRCCS Don C Gnocchi Foundation, Via Capecelatro 66, Milan 20148 , Italy; raffaello.nemni@unimi.it

Received

27 December 2002

Accepted in revised form 14 March 2003

\begin{abstract}
Objectives: Hepatitis $\mathrm{C}$ virus (HCV) infection is often associated with cryoglobulinaemia (CG). Peripheral neuropathy (PN) is a comparatively common complication of CG associated with HCV infection and it is thought to be attributable to nerve ischaemia. Only few HCV CG patients with PN have been reported. The recent finding of HCV RNA in nerve biopsy specimens has suggested a possible direct role of HCV in the pathogenesis of PN. The authors studied $51 \mathrm{HCV}$ patients to determine the prevalence of CG and to clarify the possible mechanism by which HCV determines the PN.

Methods: All the patients were studied clinically, by laboratory tests and electrophysiologically. Twenty eight patients underwent sural nerve biopsy where both morphological and morphometric evaluation of the biopsy specimen was performed, as well as statistical analysis.

Results: CG was found in 40 of 51 cases (78\%). Polyneuropathy was significantly prevalent in CG+ patients compared with CG- (18 of 40 compared with 1 of 11 patients; $p=0.01)$. HCV CG- patients more frequently developed well defined mononeuropathy or multiple neuropathy when compared with HCV CG+ (10 of 11 compared with 22 of 40; $<<0.03)$. HCV CG+ patients showed significantly higher proportion of rheumatoid factor positivity $(p<0.001)$ and low C4 levels $(p=0.001)$. Nerve biopsy was performed in 25 of $40 \mathrm{HCV} \mathrm{CG}+$ patients and in 3 of $11 \mathrm{HCV} \mathrm{CG-patients:} \mathrm{epineurial}$

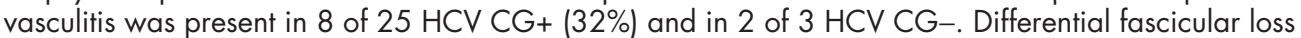
of axons was found in 10 of $25 \mathrm{CG}+(40 \%)$ and 1 of $3 \mathrm{CG}_{-}$, signs of both demyelination and axonal degeneration were present in 7 of 25 CG+ $(28 \%)$. No significant difference was found in neuropathological features, while histometrical analysis disclosed more severe involvement in CG+ patients.

Conclusions: These findings suggest that the presence of $C G$ is a negative predictive factor for the associated PN. Morphological findings in the sural nerve from HCV CG- and CG+ are consistent with an ischaemic mechanism of nerve damage and are against a direct role of the virus in causing the associated PN.
\end{abstract}


Table 1 Clinical, serological, and neuropathological data of $51 \mathrm{HCV}$ patients

\begin{tabular}{|c|c|c|}
\hline & CG+ patients & CG- patients \\
\hline Number & 40 & 11 \\
\hline Sex & $20 \mathrm{M} ; 20 \mathrm{~F}$ & $5 \mathrm{M} ; 6 \mathrm{~F}$ \\
\hline Age (y (mean (SD)) & $61(10)$ & $62(10)$ \\
\hline \multicolumn{3}{|l|}{ Clinical data (all patients) } \\
\hline Polyneuropathy, n (\%) & $18(45) \S$ & $1(9)$ \\
\hline Mononeuritis multiplex, $\mathrm{n}(\%)$ & $16(40)$ & $4(36)$ \\
\hline Cranial neuropathy, n (\%) & $3(7.5)$ & $5(46) \S$ \\
\hline Polyneuropathy + cranial neuropathy, n (\%) & $3(7.5)$ & $1(9)$ \\
\hline \multicolumn{3}{|l|}{ Laboratory data (all patients) } \\
\hline Rheumatoid factor positive, n (\%) & $35(87.5)^{*}$ & $2(18)$ \\
\hline Low C4 levels, n (\%) & $37(92.5)^{* *}$ & $5(45.5)$ \\
\hline Transaminase activity increase, $\mathrm{n}(\%)$ & $27(67.5)$ & $5(45.5)$ \\
\hline $\begin{array}{l}\text { Morphological data (number of biopsies } \\
\text { performed) }\end{array}$ & 25 & 3 \\
\hline Epineurial vasculitis, n (\%) & $8(32)$ & $2(66)$ \\
\hline Differential fascicular loss of axons, $n(\%)$ & $10(40)$ & $1(33)$ \\
\hline Demyelination + axonal degeneration, $\mathrm{n}(\%)$ & $7(28)$ & 0 \\
\hline
\end{tabular}

for IgM rheumatoid factor, C4 values, autoantobodies), detection and characterisation of cryoglobulins according to Brouet et al. ${ }^{1}$ Cryoglobulin determinations were performed at least three times during observation.

\section{Electrophysiological studies}

Electrodiagnostic tests were performed with standard electromyographic equipment (Medelec MS20 Mystro); all nerve conduction studies were carried out at a constant cutaneous temperature of $33^{\circ} \mathrm{C}$ under automatic control with a DISA type $15 \mathrm{H} 0.2$ temperature regulator system.

We studied sensory conduction of the right median and ulnar nerve and of the sural nerve of both sides in all patients. For each nerve were considered sensory conduction velocity (SCV) and sensory action potential (SAP) amplitude. Sural and ulnar SAPs were recorded antidromically at the ankle and wrist respectively, whereas SAPs of the median nerve were obtained by antidromical stimulation at wrist and elbow to determine separately wrist to finger and elbow to wrist SCV. Computer averaging was used to determine the size of low amplitude sensory responses.

We also studied motor conduction of median, ulnar and deep peroneal nerves; for each nerve we evaluated motor conduction velocity $(\mathrm{MCV})$, compound motor action potential (CMAP) amplitude and distal latency (DL). To detect changes of proximal conduction we recorded $F$ waves (20 times for each test) from ulnar and deep peroneal nerve at the elbow and above the knee respectively: the shortest F wave latency was considered.

Muscles that underwent EMG needle examination were tibialis anterior and medial gastrocnemius. Electrophysiological parameters of the patients were considered abnormal if outside twice the standard deviation of mean values obtained from healthy age matched controls.

\section{Neuropathological studies}

We studied 28 sural nerve biopsy specimens, 25 from PN CG + and three from PN CG- patients.

The sural nerve biopsy specimens were taken just proximal to the lateral malleolus. A portion was fixed in $10 \%$ formalin, embedded in paraffin wax, and sections were stained with haematoxylin and eosin, Congo red, alcian blue, and PAS. A second portion was quickly frozen in liquid nitrogen and either frozen or paraffin wax embedded; $6 \mu \mathrm{m}$ sections were used for direct immunocytochemical study using peroxydase conjugated goat antihuman affinity purified antibodies (IgG, IgA, IgM, 1:500 diluitions). A third portion was fixed in $2 \%$ buffered glutaraldehyde, postfixed in $1 \%$ osmium tetroxide, dehydrated, and embedded in Epon 812. Semithin transverse sections were stained with toluidine blue and examined under the light microscope to estimate the distribuition and severity of myelinated fibre abnormalities. Quantitative histometrical analysis of myelinated fibres were obtained from micrographs, at a magnification of $\times 1000$ of three fascicles for each patient. Fifty teased myelinated fibres were studied in each patient.

The sections were analysed for the presence of signs of axonal degenerations or demyelination. Particular attention was focused on the presence of perivascular and interstitial inflammatory infiltrates; other vascular abnormalities, such as hyperplasia of muscular and endothelial cell layers, were noted. Epineurial vasculitis was diagnosed according to the criteria of Dyck et al ${ }^{19}$ when intramural infiltration with necrosis of the vessel walls was present.

\section{Statistical methods}

Statistical analysis of the results were performed using a $\chi^{2}$ test (associated to Yates's correction when necessary) and Student's $t$ test for unpaired data. p Values $<0.01$ and $<0.05$ were considered significant.

\section{RESULTS}

Fifty one HCV infected patients, 25 men and 26 women, were studied. Table 1 summarises their clinical, laboratory, and neuropathological features. Their ages at time of diagnosis ranged from 39 to 81 years; mean (SD) age 62 (10). Detectable cryoglobulins (with a cryocrit greater than $0.1 \%$ ) were found in the serum in 40 of 51 patients (78\%). Cryoglobulins were type III and type II. No patient had type I cryoglobulins.

Clinical examination disclosed four different patterns of peripheral nerve involvement: PN, mononeuropathy and/or multiple neuropathy $(\mathrm{MN})$, cranial neuropathy $(\mathrm{CN})$, polyneuropathy combined with cranial neuropathy $(\mathrm{PN}+\mathrm{CN})$. PN was a symmetrical sensorymotor neuropathy with predominant sensory features in some cases. Subgroups of multiple mononeuropathy and cranial neuropathy as well as cranial neuropathy associated with clinical polyneuropathy, were all considered to be clinical expression of an ischaemic nerve damage as other causes of focal nerve damage were excluded by inclusion clinical criteria. 
Table 2 Motor and sensory nerve conduction studies in CG+ and CG-patients (mean (SD))

\begin{tabular}{|c|c|c|c|}
\hline & Controls & CG+ & CG- \\
\hline \multicolumn{4}{|l|}{ Deep peroneal nerve } \\
\hline $\mathrm{MCV}(\mathrm{m} / \mathrm{sec})$ & $51.5(3.4)$ & $42.1(7.3)^{*}$ & $48.0(3.9)$ \\
\hline $\mathrm{DL}$ (msec) & $4.1(0.5)$ & $5.1(1.3)$ & $5.0(1.1)$ \\
\hline $\mathrm{Amp}(\mathrm{mV})$ & $11.5(3.7)$ & $4.8(3.5)$ & $7.5(4.2)$ \\
\hline $\mathrm{F}$ wave (msec) & $40.5(2.6)$ & $44.3(5.0)$ & $40.6(5.0)$ \\
\hline \multicolumn{4}{|l|}{ Median nerve } \\
\hline $\mathrm{MCV}(\mathrm{m} / \mathrm{sec})$ & $58.1(3.3)$ & $55.5(3.4)$ & $55.5(3.3)$ \\
\hline $\mathrm{DL}$ (msec) & $3.4(0.3)$ & $3.5(0.3)$ & $3.8(0.4)$ \\
\hline $\mathrm{Amp}(\mathrm{mV})$ & $17.0(3.9)$ & $20.2(5.2)$ & $16.8(4.1)$ \\
\hline \multicolumn{4}{|l|}{ Ulnar nerve } \\
\hline $\mathrm{MCV}(\mathrm{m} / \mathrm{sec})$ & $59.6(4.2)$ & $57.9(4.1)$ & $58.4(4.5)$ \\
\hline $\mathrm{DL}$ (msec) & $2.5(0.3)$ & $2.8(0.3)$ & $2.6(0.2)$ \\
\hline $\mathrm{Amp}(\mathrm{mV})$ & $16.8(2.5)$ & $14.8(3.1)$ & $15.1(2.1)$ \\
\hline F wave (msec) & $22.3(0.2)$ & 23.7 (1.9) & $23.2(1.2)$ \\
\hline \multicolumn{4}{|l|}{ Sural nerve } \\
\hline $\mathrm{SCV}(\mathrm{m} / \mathrm{sec})$ & 50.8 (3.7) & $42.9(14.3)$ & 43.3 (14.3) \\
\hline SAP ampl (mcV) & $22.9(12.2)$ & $6.0(5.8)$ & 10.9 (11.1) \\
\hline \multicolumn{4}{|l|}{ Median nerve } \\
\hline W-F SCV $(\mathrm{m} / \mathrm{sec})$ & $56.7(3.2)$ & $51.6(5.7)$ & $50.2(4.0)$ \\
\hline SAP ampl. (mcV) & $39.3(20.8)$ & $28.3(8.6)$ & 34.5 (21.0) \\
\hline E-W SCV $(\mathrm{m} / \mathrm{sec})$ & $62.7(3.2)$ & $58.2(4.9)$ & $60.3(4.2)$ \\
\hline SAP ampl (mcV) & 24.4 (17.9) & $12.0(5.1)$ & 17.9 (11.0) \\
\hline \multicolumn{4}{|l|}{ Ulnar nerve } \\
\hline $\mathrm{SCV}(\mathrm{m} / \mathrm{sec})$ & $55.6(4.6)$ & $51.7(4.2)$ & $50.5(3.7)$ \\
\hline SAP ampl (mcV) & $35.0(18.8)$ & $28.8(11.2)$ & $37.6(24.9)$ \\
\hline
\end{tabular}

Of the 40 CG+ patients, 18 had PN (45\%), 16 had MN $(40 \%), 3$ had $\mathrm{CN}(7.5 \%)$, and 3 had combined PN +CN (7.5\%). Among the 11 CG- patients, the clinical features were as follows: five patients had CN (46\%), four patients had MN (36\%), one patient had PN (9\%), and one patient had combined $\mathrm{PN}+\mathrm{CN}(9 \%)$ (table 1$)$.

The prevalence of PN was significantly higher in CG+ patients compared with CG- ( $45 \%$ versus $9 \% ; p=0.01, \chi^{2}$ test $)$. CN was significantly higher in CG- patients (46\% versus $7.5 \%$; $\mathrm{p}=0.01, \chi^{2}$ test). There was no significant difference in the proportion of mononeuropathy/ multiple neuropathy and cranial neuropathy combined to polyneuropathy.

Considering $\mathrm{CN}, \mathrm{MN}$, and $\mathrm{CN}+\mathrm{PN}$ as expression of a mononeuritic process, we found a significant difference between $C G+$ and $C G-$ groups $\left(p<0.03, \chi^{2}\right.$ test $)$ : $C G-$ patients more frequently developed a well defined mononeuritic process ( 10 of $11,90 \%$ ) when compared with CG + patients (22 of $40,55 \%)$.

CG+ patients showed significantly higher proportion with rheumatoid factor positivity $\left(87.5 \%\right.$ versus $18 \%$; $>0.001, \chi^{2}$ test) and lower C4 levels (92.5\% versus $45.5 \% ; \mathrm{p}=0.001, \chi^{2}$ test). Increased transaminase activities (ALT $>70 \mathrm{U} / 1)$, as possible expression of HCV cytopathic effect, were found in $67.5 \%$ of CG+ patients compared with $45.5 \%$ of CG- patients; no significant difference was found between the two groups $\left(\chi^{2}\right.$ test).

Table 2 gives the electrophysiological findings. Analysis of the data showed a significant difference only for one parameter: MCV of deep peroneal nerve in CG+ compared

Table 3 Histometry of sural nerve biopsy specimens (mean (SD))

\begin{tabular}{llll}
\hline & Controls & CG + & CG- \\
\hline Fibre density $\left(\mathrm{n} / \mathrm{mm}^{2}\right)$ & $5.400-$ & 2.357 & 4.210 \\
& 8.600 & $(285)^{*}$ & $(964)$ \\
Fibres $>8 \mu \mathrm{m}(\%)$ & $22.8-34.6$ & $9.6(4.9)^{* *}$ & $20.7(7.2)$ \\
Total number of clusters & $<38$ & $17.5(5.3)$ & $99.4(45)^{*}$ \\
\hline${ }^{*} \mathrm{p}<0.005 ;{ }^{*} \mathrm{*}<0.05$ (Student's + test for unpaired groups).
\end{tabular}

with CG- patients. The other neurophysiological parameters were suggestive of a wider and more severe involvement of peripheral nerve in CG + patients, even if no significant differences were found.

Nerve biopsy was performed in 25 CG + patients: epineurial vasculitis (fig 1 ) was present in 8 of 25 cases (32\%), differential fascicular loss of axons (fig 2) was found in 10 cases $(40 \%)$, signs of both demyelination and axonal degeneration were present in seven cases $(28 \%)$. Three of the 11 CGpatients underwent sural nerve biopsy: two patients had epineurial vasculitis and one showed a differential fascicular loss of axons. In all cases, teased fibre preparation demonstrated axonal degeneration without evidence of primary demyelination. Comparison of neuropathological features disclosed no significant difference between CG + and CG- groups $\left(\chi^{2}\right.$ test). Table 3 gives the histometrical data of sural nerve biopsy specimens. We found a significant fibre loss in CG+ compared with $\mathrm{CG}-$ patients $(\mathrm{p}<0.005, t$ test $)$, as well as in

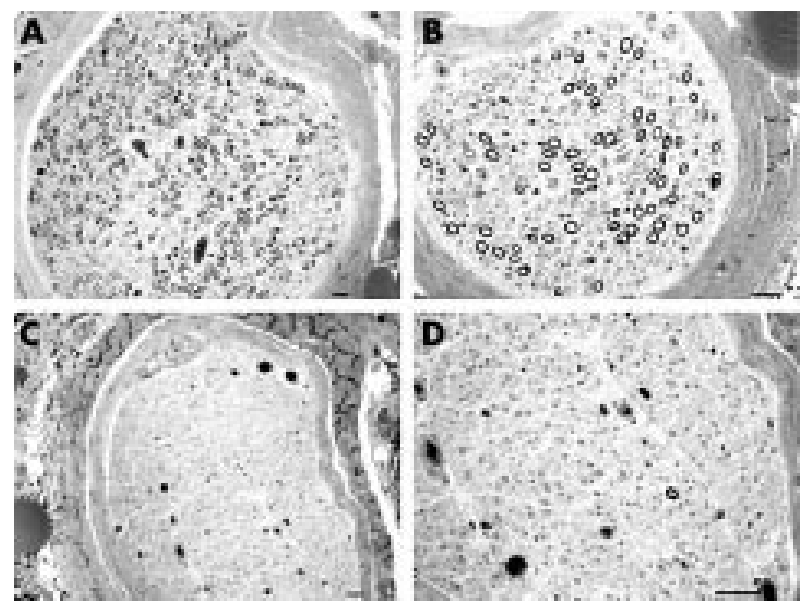

Figure 1 Transverse section of sural nerve. The non-uniform pattern of myelinated fibre loss is shown in four different fascicles (A, B, C, and D) from the same CG+ patient. (Epon embedded semithin section, toluidine blue. Bar $=20 \mu \mathrm{m}$.) 

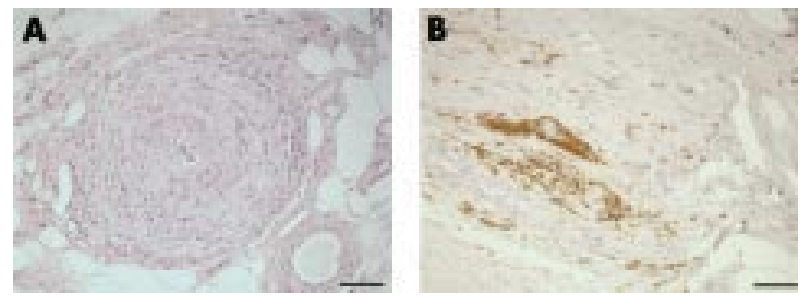

Figure 2 (A) Transverse section of sural nerve of a CG- patient. Vasculitis of small sized artery in the epineurium: inflammatory infiltration of the vessel wall and perivascular space is present. (Paraffin wax embedded section, haematoxylin and eosin. Bar=50 um.) (B) Immunostaining demonstrates that inflammatory cells are lymphocytes. (Cryosection stained with peroxidase conjugated antibodies to lymphocytes. Bar=50 $\mathrm{mm}$.)

percentage of large myelinated fibres $(\mathrm{p}<0.05, t$ test $)$. Total number of clusters, representing regenerating fibres, was significantly increased in CG- compared with CG + patients $(\mathrm{p}<0.005, t$ test $)$.

\section{DISCUSSION}

The association between HCV infection and CG is well established. Detectable CG are present in about $50 \%$ of HCV patients although most of them do not have CG related symptoms. ${ }^{23}$ A sensory motor PN has been found in up to $9 \%$ of patients chronically infected with $\mathrm{HCV}^{2021}$ and the prevalence rises up to $30 \%$ in HCV CG + patients. $^{78}$ Most data in the literature are concerned with neuropathy in HCV CG+ patients, whereas only few of them investigate neuropathy in HCV CG- patients. ${ }^{16}{ }^{17}$ Several previous studies characterised PN involvement in HCV CG+ patients as a subacute, distal, motor sensory polyneuropathy. An asymmetrical sensory impairment has often been highlighted..$^{722}$ Mono and multiple mononeuritis have also been frequently reported. ${ }^{53}$ Surveys on HCV CG- patients are few, based on small samples and lacking clinical and neuropathological data.

In our series of patients affected by HCV infection and neuropathy, CG were found in $78 \%$ of patients. Polyneuropathy was significantly prevalent in CG+ patients, whereas CGpatients showed a higher prevalence of mononeuropathy or multiple neuropathy. Also Lidove, ${ }^{16}$ in his small series, reported a high prevalence of mono or multiple neuropathy in HCV CG- patients (three of four patients). As a matter of fact, electrophysiological analysis disclosed a wider than expected peripheral nervous system involvement, also in HCV CGpatients with clinical mono or multiple neuropathy. These data seem to suggest a different degree of the same pathological mechanism of nerve damage, ranging from mild/moderate in HCV CG- patients to moderate/severe in HCV CG + patients. Laboratory data disclosed a prevalence of RF+ and lower C4 levels in HCV CG + as compared with HCV CG-, confirming data previously reported in the literature ${ }^{821}$ and indicating complement activation and consumption, caused by immunocomplex formation. On the other hand, most HCV CGpatients had RF negativity and normal C4 values, as well as the four HCV CG- patients described by Lidove. ${ }^{16}$ Nevertheless, some HCV CG- patients in our series, showed humoral signs of complement activation indicating that this process was not necessarily related to the presence of CG.

Peripheral neuropathy associated with HCV is mainly characterised by axonal damage and it is usually associated with CG. It was postulated that nerve damage is secondary to epineurial vessels changes caused by occlusion or vasculitis induced by longstanding cryoglobulin precipitation with complement fixation and RF deposition. The vasculitis or vascular occlusion causes fascicular ischaemia that results in axonal degeneration. ${ }^{11} 1319$

It has been supposed that HCV may have a direct role in the pathogenesis of neuropathy; it could induce nerve damage by a direct cytopathic effect or by an immunomediated mechanism such as immune complex induced changes of the epineural vessels. This hypothesis seems to be supported by the finding of HCV RNA in five nerve biopsy specimens by RT-PCR in $\operatorname{situ}^{24}$ and could explain the neurological involvement in CG- patients. It has also been supposed that CG or immunocomplexes, or both, contribute to the generation of microvascular, but not vasculitic, changes in epineurial and endoneurial capillaries, whereas only T cell dependent mechanism account for the epineurial inflammation. ${ }^{18}$

Only isolated reports are available regarding neuromuscular biopsies in CG- patients ${ }^{16}{ }^{17}$ disclosing mainly axonal damage without demonstration of a vasculitic process.

We found pathological evidence of a vasculitic process both in HCV CG + and in HCV CG- patients. In fact either epineurial vasculitis and fascicular axonal loss suggest ischaemia as a cause of the neuropathy.

Cryoglobulins are immunocomplexes able to activate the complement pathway and cause vasculitis. Regarding the pathophysiology of the vasculitic process in HCV CGneuropathy, the activation of the complement may be attributable to three different mechanisms: an innate mechanism caused by the ability of the virus itself to activate the complement pathway, a second mechanism based on the reactivity of natural killer cells against the viral proteins, and a third mechanism supposing the existence of an interaction between HCV and anti-HCV antibodies. Thus, vasculitis might be attributable to immunocomplex deposition not related to the presence of CG in the serum. It is well established that the major HCV envelope protein inhibits natural killer cells ${ }^{25}$; nevertheless complement pathway may be activated by the reactivity of natural killer cells with HCV or by HCV/anti-HCV immunocomplex formation. To date there are no data in the literature concerning the ability of HCV to activate the innate mechanism of complement activation, even if it is well known that virus may trigger complement pathway independently of adaptive immune responses. ${ }^{26}$

In summary, our study disclosed a more severe impairment in HCV CG + patients as compared with CG-, either by clinical, electrophysiological, and histometrical analysis. The mechanism of peripheral nerve damage seems to be vasculitic in both CG + and CG- patients, as supported by the clinical and morphological findings. The presence of CG in the serum is predictive of a more severe and widespread neuropathic involvement, but there is evidence that cryoglobulins are not the unique factor involved in the vasculitic process. Further studies are needed to clarify the role of HCV in innate complement activation and the relevance of the "in situ" HCV induced chronic stimulation of the immune system.

\section{Authors' affiliations}

R Nemni, L Sanvito, G Santuccio, N Canal, Department of Neurorehabilitation, IRCCS Don C Gnocchi Foundation, University of Milan, Italy

A Quattrini, Department of Neurology, IRCCS S Raffaele, Milan, Italy M Camerlingo, Department of Neurology, Ospedali Riuniti, Bergamo, Italy

Competing interests: none declared.

\section{REFERENCES}

1 Brouet JC, Clauvel JP, Danon F, et al. Biologic and clinical significance of cryoglobulins: a report of 86 cases. Am J Med 1974;57:775-88.

2 Adinolfi LE, Utili R, Attanasio V, et al. Epidemiology, clinical spectrum and prognostic value of mixed cryoglobulinaemia in hepatitis $C$ virus patients: a prospective study. It J Gastroenterol 1996;28:1-9.

3 Lunel F, Musset L, Cacoub P, et al. Cryoglobulinemia in chronic liver disease: role of hepatitis $C$ virus and liver damage. Gastroenterology 1994; 106:1291-300.

4 Ferri C, Greco F, Longombardo G, et al. Antibodies to hepatitis $C$ virus in patients with mixed cryoglobulinemia. Arthritis Rheum $1991 ; 34: 1606-10$ 
5 Gemignani F, Pavesi G, Fiocchi A, et al. Peripheral neuropathy in essential mixed cryoglobulinemia. J Neurol Neurosurg Psychiatry 1992;55: $116-20$.

6 Logothetis J, Kennedy WR, Ellington A, et al. Cryoglobulinemic neuropathy. Incidence and clinical characteristics. Arch Neurol 1968:19:389-97.

7 Migliaresi S, Di iorio G, Ammendola A, et al. [Peripheral nervous system involvement in HCV-realted mixed cryoglobulinemia]. [In Italian]. Reumatismo 2001:53:26-32.

8 Zaltron S, Puoti $M$, Liberini $P$, et al. High prevalence of peripheral neuropathy in hepatitis $C$ virus infected patients with symptomatic and asymptomatic cryoglobulinemia. It J Gastroenterol Hepatol 1998;30:391-5

9 Cavaletti G, Petruccioli MG, Crespi V, et al. A clinico-pathological and follow-up study of 10 cases of essential type II cryoglobulinemic neuropathy. J Neurol Neurosurg Psychiat 1990;53:586-9.

10 Garcia-Bragado F, Fernandez JM, Navarro C, et al. Peripheral neuropathy in essential mixed cryoglobulinemia. Arch Neurol 1988;45:1210-14

11 Nemni R, Corbo M, Fazio R, et al. Cryoglobulinaemic neuropathy: a clinical, morphological and immunocytochemical study of 8 cases. Brain $1988 ; 111: 541-52$

12 Vital C, Deminiere C, Lagueny A, et al. Peripheral neuropathy with essential mixed cryoglobulinemia: biopsies from five cases. Acta Neuropathol 1988:75:605-10.

13 Chad D, Pariser K, Bradley WG, et al. The pathogenesis of cryoglobulinemic neuropathy. Neurology 1982;32:725-9.

14 Vallat JM, Desproges-Gotteron R, Leboutet $\mathrm{W}$, et al. Cryoglobulinemic neuropathy: a pathological study. Ann Neurol 1980;8:179-85.

15 Betourné $C$, Buge $A$, Dechy $H$, et al. Neuropathies périphériques au cours d'un myélome ${ }^{\circ} \mathrm{lgA}$ et d'une cryoglobulinémie mixte: traitment par plasmaphérèses itératives. Nouvelle Presse Médicale 1980;9:1369-71.
16 Lidove O, Cacoub P, Maisonobe T, et al. Hepatitis $C$ virus infection with peripheral neuropathy is not always associated with cryoglobulinaemia. Ann Rheum Dis 2001;60:290-2.

17 Paoletti V, Donnarumma L, De Matteis A, et al. Peripheral neuropathy without cryoglobulinemia in patients with hepatitis $C$ virus infection. Panminerva Med 2000;42:175-8.

18 Bonetti B, Invernizzi F, Rizzato N, et al. T-cell-mediated epineurial vasculitis and humoral-mediated microangiopathy in cryoglobulinemic neuropathy. J Neuroimmunol 1997:73:145-54.

19 Dyck PJ, Benstead TJ, Conn DL, et al. Non systemic vasculitic neuropathy. Brain 1987;110:843-54.

20 Cacoub $\mathbf{P}$, Renou , Rosenthal E, et al. Extrahepatic manifestations of hepatitis $C$ virus infection in 321 patients. Medicine (Baltimore) 2000;79:47-56

21 Trejo O, Ramos-Casals M, Garcia-Carrasco M, et al. Cryoglobulinemia. Study of etiologic factors and clinical and immunologic features in 443 patients from a single center. Medicine 2001;80:252-62.

22 Gemignani F, Melli G, Inglese C, et al. Cryoglobulinemia is a frequent cause of peripheral neuropathy in undiagnosed referral patients. $J$ Peripher Nerv Syst 2002;7:59-64.

23 Apartis E, Léger JM, Musset L, et al. Peripheral neuropathy associated with essential mixed cryoglobulinemia: a role for hepatitis $C$ virus infection? J Neurol Neurosurg Psychiat 1996;60:661-6.

24 Bonetti B, Scardoni M, Monaco S, et al. Hepatitis $C$ virus infection of peripheral nerves in type II cryoglobulinaemia. Virchows Arch 1999:434:533-5.

25 Crotta S, Stilla A, Wack A, et al. Inhibition of natural killer cells through engagement of CD81 by the major hepatitis $C$ virus envelope protein. $J$ Exp Med 2002; 195:35-41.

26 Walport MJ. Complement. First of two parts. N Engl J Med 2001;344: 1058-66.

\section{NEURONLINE}

\section{Pubcrawler: www.pubcrawler.ie}

A ll of us suffer from significant difficulty in keeping up to speed with the neurological literature. One study suggested that a dedicated doctor would have to read no less than 17 scientific articles a day, in order to keep pace with the medical literature. Quite simply having the time to keep up to date with your own area of subspecialist interest is a significant issue. One solution to this very real clinical problem is a website called PubCrawler (www.pubcrawler.ie). PubCrawler is a free "alerting" service set up by the genetics department of Trinity College, Dublin, that is specifically used for searching through publicly available databases such as Medline and Genbank. Genbank is not exactly the kind of database that the average general neurologist would want to trawl through, but Medline obviously is. The user can set the Medline criteria that one wants to search under, and PubCrawler will email you to notify of any updates in the literature at a preset time. I have used PubCrawler for over 4 years now, I have it set up to perform searches on Parkinson's disease, multiple system atrophy, and progressive supranuclear palsy on a weekly basis. On a typical week, I will be emailed about 40 citations, which I can quickly skim through (in less than 5 minutes), and select the abstracts that are of specific interest to me.

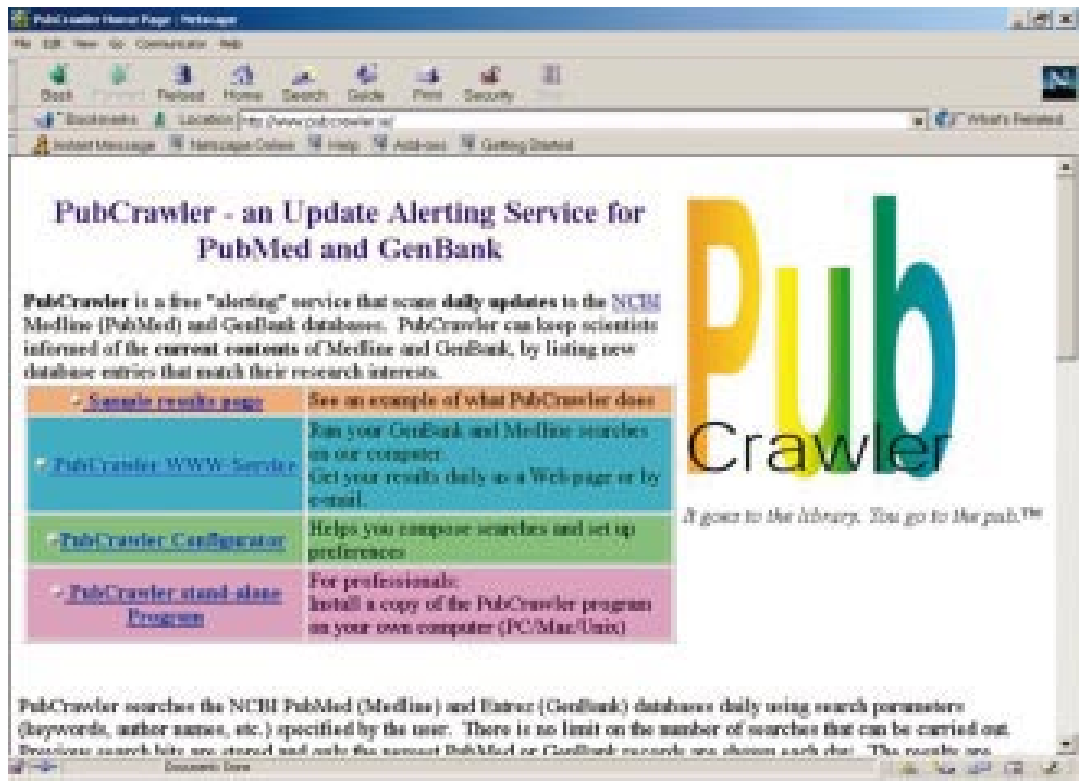

One criticism relates to the graphical look of the website, which is not particularly appealing, and the user will need to be confident in their use of Medline search criteria. However, these are minor issues, this really is a website that I would strongly recommend to any clinician or neuroscientist. The PubCrawler system may not be perfect (although it comes close), but it must have saved me hours of work in the library. As the website's logo affectionately states "It goes to the library, you go to the pub".

D Nicholl

Department of Neurology, Queen Elizabeth d.j.nicholl@bham.ac.uk Hospital, Birmingham B15 2TH, UK; 